The Astrophysical Journal, 180: 351-358, 1973 March 1

(C) 1973. The American Astronomical Society. All rights reserved. Printed in U.S.A.

\title{
OPTICAL AND NEAR-INFRARED OBSERVATIONS OF THE NEARBY SPIRAL GALAXY MAFFEI 2
}

\author{
Hyron Spinrad,* J. Bahcall, $\dagger$ E. E. Becklin, $\ddagger$ James E. Gunn $₫$ J. Kristian, $\S$ \\ G. Neugebauer, $\ddagger$ W. L. W. Sargent, $\ddagger$ and H. Smith* \\ Received 1972 August 7
}

\section{ABSTRACT}

Spectra, photographs, and photometric measurements have been used to show that Maffei 2 has a distance of $5 \pm 2 \mathrm{Mpc}$ and that it has a morphological type near Sbc II-in agreement with similar conclusions made earlier by radio observers.

We discuss the possible relationships between Maffei 2 and the elliptical galaxy Maffei 1; there are serious inconsistencies in the existing data which bear on this question.

Subject headings: galaxies, individual — infrared sources

\section{INTRODUCTION}

The recent identification of the two heavily obscured nearby galaxies Maffei 1 and 2 (cf. Maffei 1968) by Spinrad et al. (1971) has been followed by a new program of near-infrared photography and photometry, and optical spectroscopy and photometry which we describe here. Here we have concentrated on Maffei 2, the system largely ignored by Spinrad et al. (1971). We show that this object is a medium-sized, averageluminosity, spiral galaxy of type $\mathrm{Sc}$ or $\mathrm{Sbc}$ - a result which has already been indirectly suggested on other grounds by various radio observers (Bottinelli et al. 1971; Shostak and Weliachew 1971; Love 1972; Allen and Raimond 1972). In addition we discuss the information on the reddening, distance, and stellar content of the nucleus of Maffei 2 which is provided by our new observations.

\section{OBSERVATIONS}

\section{a) Direct Photography}

Gunn obtained a direct photograph of the inner disk and the nucleus of Maffei 2 at the prime focus of the Hale 200 -inch $(508-\mathrm{cm})$ reflector which is shown in figure 1 (plate 4). The detector was a Wratten 89B filtered ITT 4708 image intensifier whose phosphor was photographed with a IIa-D plate; this combination gives an effective wavelength of $\lambda=8500 \AA$ for a red object. Note the sharp nucleus, the extended disk, and the narrow dust lanes parallel to the major axis of the system. The inner part of Maffei 2 is similar to the best short-exposure photographs of the inner disk of M31 which also exhibits narrow dust lanes and patches. Maffei 2 also shows some hints of inner spiral structure on the east side of the system.

Kristian, Bahcall, and Spinrad obtained both medium and heavily exposed 48-inch $(122-\mathrm{cm})$ Schmidt reflector plates in 1969 and 1970. They were taken on $\mathrm{I}^{-\mathrm{N}^{+}}$emulsion behind a Wratten 89B filter; the hypersensitization technique was described by Spinrad

* University of California at Berkeley.

$\dagger$ Division of Physics, Mathematics, and Astronomy, California Institute of Technology. Present address: Institute for Advanced Study, Princeton, New Jersey.

\$Division of Physics, Mathematics, and Astronomy, California Institute of Technology; Hale Observatories, California Institute of Technology, Carnegie Institution of Washington.

$\S$ Hale Observatories, Carnegie Institution of Washington, California Institute of Technology. 
(1966). On figure 2 (plate 5) of $3^{\mathrm{h}} 24^{\mathrm{m}}$ exposure, Maffei 2 shows a barred inner structure of about $5^{\prime}$ length on the major axis and two weak but clearly visible spiral arms, the strongest extending southwest of the Maffei 2 central region. The major-axis extent is approximately $11^{\prime}$; this diameter measurement is limited by the substantial background fog on the deep plate. This fog is partly due to ammoniation, but is mainly due to the strong airglow, largely $\mathrm{OH}$, present in the near-infrared sky spectrum. The spiral structure, especially a short "spur" almost perpendicular to the southwest part of the Maffei 2 "bar," is also clearly visible on the Schmidt plate prints.

Maffei 1, described by Spinrad et al. (1971) as a large E galaxy, can be seen in figure 2 as an E3-shaped system some 45' west of Maffei 2. The outer wings of Maffei 1 may be traced to about $7^{\prime}$ radius on the deepest exposure. The north side of Maffei 1 is more heavily covered with dust than the other parts of the easily visible central portion of the galaxy; we cannot say to which galaxy this dust actually belongs.

An $\mathrm{H} \alpha$ photograph of Maffei 2 obtained by Spinrad on 098-O2 emulsion with a RG2 filter at the prime focus of the Lick 120-inch $(305-\mathrm{cm})$ telescope is shown in figure 3 (plate 6). This exposure shows that many of the knots visible in the other figures are clearly $\mathrm{H}$ II regions; particularly prominent ones are marked in figure 3.

It is hard to classify Maffei 2 on these photographs because of the heavy foreground obscuration; however, a morphological type near Sbc II is a reasonable compromise. The galaxy apparently has a weakly barred structure. Maffei 2 thus appears similar to or "later than" M31, as the radio observations have already indicated. Based on figure 3, the inclination angle of Maffei 2 is about $55^{\circ}$.

\section{b) Spectra}

Two spectrograms of Maffei 2 were obtained by Sargent with the Cassegrain imagetube spectrograph on the Hale 200-inch telescope. The spectrograms have a dispersion of $180 \AA \mathrm{mm}^{-1}$ and cover the wavelength range $3800-6800 \AA$ in reasonable focus. The spectrum of the night sky is very strong on each spectrogram; in only one of them is there a very faint red continuum, extending about $10^{\prime \prime}$ along the slit, which is presumably due to the stars in the center of Maffei 2 . In addition, each spectrogram shows a faint emission line, also coming from a region about $10^{\prime \prime}$ in extent along the slit, with a wavelength of $6584.1 \AA$. A much fainter emission line, also extending about $10^{\prime \prime}$ along the slit, and barely detectable on these spectrograms, has a wavelength of $6560.9 \AA$. The most obvious identification of these emission features is that they are [N II] $\lambda 6583.6$ and $\mathrm{H} \alpha \lambda 6562.8$, respectively. The resulting radial velocities are $+22 \mathrm{~km} \mathrm{~s}^{-1}$ from [N II] $\lambda 6583.6$ and $-59 \mathrm{~km} \mathrm{~s}^{-1}$ from $\mathrm{H} \alpha$. The straight mean radial velocity is $-18 \mathrm{~km} \mathrm{~s}^{-1}$; a more reasonable value obtained by weighting [N II] and $\mathrm{H} \alpha$ in the ratio $3: 1$, to allow roughly for their difference in intensity, would lead to $V_{R}=+1 \mathrm{~km} \mathrm{~s}^{-1}$.

The results of these spectroscopic measurements should be treated with the utmost caution. Since Maffei 2 is in the galactic plane, it is possible that the measured features arise in a diffuse foreground $\mathrm{H}$ II region in our Galaxy. The fact that the nuclei of $\mathrm{Sa}$ and $\mathrm{Sb}$ galaxies frequently have spectra in which [N II] is stronger than $\mathrm{H} \alpha$ should only be taken as very weak confirmation that the emission lines reported here actually arise in Maffei 2 . The fact that the $21-\mathrm{cm}$ observations also lead to a systemic radial velocity near zero is slightly stronger confirmation.

\section{c) Optical Photometry}

Continuum scans of Maffei 2 have been made by Spinrad and Gunn. They used the Wampler scanner on the Lick Observatory 120-inch telescope and Oke's multichannel scanner on the Hale 200-inch telescope, respectively. The Lick scans were made through an aperture $15^{\prime \prime}$ in diameter centered on the nucleus of Maffei 2; they cover 
the region $5000-10,700 \AA$. The Hale observations were made with a 14 " aperture and cover the region $3260-10,540 \AA$. The two sets of measurements agree. Details of the Hale observations, which were more extensive, are listed in table 1; the data are also plotted in figure 4.

\section{d) Infrared Photometry}

Broad-band infrared measurements of Maffei 2 have been made by Becklin and Neugebauer at the 60- and 100-inch $(152-$ and $254-\mathrm{cm})$ telescopes at Mount Wilson and the 20- and 200-inch telescopes at Palomar Mountain. The effective wavelengths at which observations were carried out were $1.65 \mu(1.5-1.8 \mu), 2.2 \mu(2.0-2.4 \mu)$, and $3.5 \mu(3.2-3.9 \mu)$; focal-plane apertures ranging from $5^{\prime \prime}$ to $135^{\prime \prime}$ diameter were used at $2.2 \mu$, while only more restricted observations were made at 1.65 and $3.5 \mu$. The flux densities obtained are shown in figure 4 as a function of aperture diameter; the

TABLE 1

Optical Photometry of Central 14.0" of Maffei 2

\begin{tabular}{|c|c|c|}
\hline$\lambda_{(\AA)}^{a)}$ & $\log f \operatorname{lux}(b)$ & $\sigma^{c)}$ \\
\hline $3260 \ldots$ & neg. flux & 0.3 . \\
\hline $3420 \ldots$ & neg. flux & 0.4 \\
\hline $3580 \ldots$ & neg. flux & 0.2 \\
\hline $3740 \ldots$ & -27.7 & 0.3 \\
\hline $3900 \ldots$ & -27.8 & 0.2 \\
\hline $4060 \ldots$ & -27.7 & 0.2 \\
\hline $4220 \ldots$ & -27.4 & 0.1 \\
\hline $4380 \ldots$ & $-27 \cdot 3$ & 0.1 \\
\hline $4540 \ldots$ & -27.3 & 0.1 \\
\hline $4700 \ldots$ & -27.03 & 0.4 \\
\hline $4680 \ldots$ & -27.04 & 0.4 \\
\hline $5020 \ldots$ & -27.78 & 0.2 \\
\hline $5780 \ldots$ & -26.60 & 0.3 \\
\hline $5340 \ldots$ & -26.49 & 0.1 \\
\hline $5500 \ldots$ & -26.37 & 0.2 \\
\hline $5660 \ldots$ & -26.32 & 0.2 \\
\hline $5860 \ldots$ & -26.16 & 0.1 \\
\hline $6220 \ldots$ & -25.95 & 0.1 \\
\hline $6580 \ldots$ & -25.73 & 0.005 \\
\hline $7300 \ldots$ & -25.38 & 0.005 \\
\hline $7680 \ldots$ & -25.24 & 0.005 \\
\hline $8020 \ldots$ & -25.09 & 0.005 \\
\hline $8380 \ldots$ & -24.91 & 0.005 \\
\hline $8740 \ldots$ & -24.79 & 0.005 \\
\hline $9100 \ldots$ & -24.64 & 0.005 \\
\hline $9460 \ldots$ & -24.54 & 0.005 \\
\hline $10180 \ldots$ & -24.41 & 0.005 \\
\hline $10540 \ldots$ & $-24 \cdot 30$ & 0.005 \\
\hline
\end{tabular}

a) The wavelength intervals were $160 \AA$ from $\lambda 3250$ through $\lambda 5660$ and $360 \AA$ from $\lambda 5860$ through $\lambda 10540$; b) The flux is in units of erg $\mathrm{cm}^{-2} \mathrm{~s}^{-1}$;

c) The errors in log flux represent the formal standard error from the counting statistics; the real uncertainties are probably not better than 0.02 in $\log$ flux. 


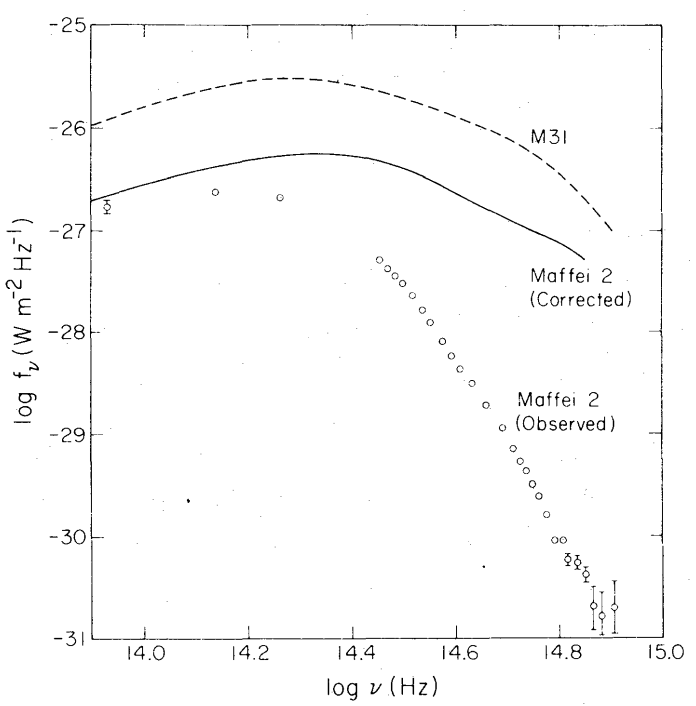

FIG. 4

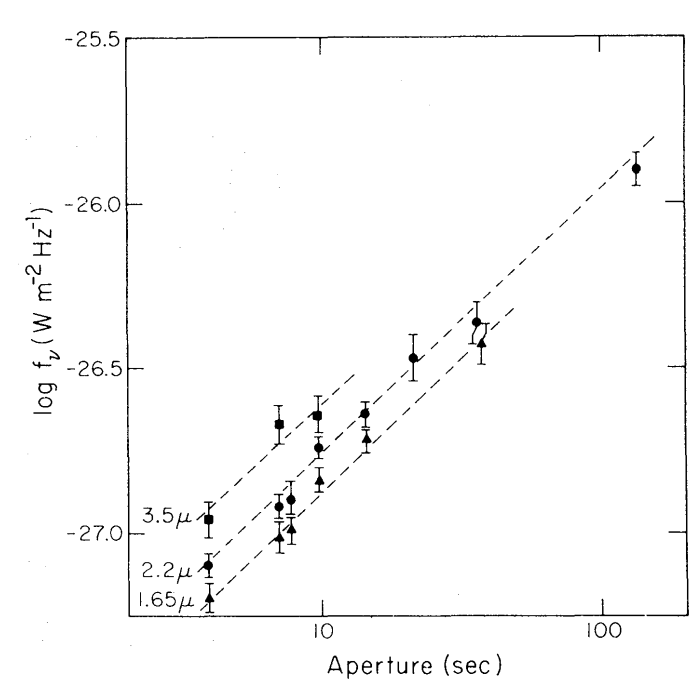

FIG. 5

FIG. 4.-The energy distribution of the central 14" of Maffei 2 obtained with the 200-inch multichannel spectrometer and the Caltech infrared photometer. The solid curve shows the energy distribution on Maffei 2 unreddened by 6.3 mag of visual extinction while the dashed curve is the energy distribution of M31 from Sandage et al. (1969).

FIG. 5.- The integrated flux within an aperture with the stated diameter is shown for 1.65-, 2.2-, and 3.5- $\mu$ radiation. The $3.5-\mu$ data have been offset by 0.50 in log flux for clarity.

measurements were centered on the visible nucleus. The photometer has been described by Becklin and Neugebauer (1968) and measures the difference between the emission from two adjacent areas of sky; the separation of the areas varied depending on the telescope and aperture used. A correction for the energy in the second "reference" beam was made by assuming that the power law exhibited by the $1.65-$ and $2.2-\mu$ data in figure 5 held for the surface brightness at the second beam. The correction was typically on the order of 10 percent.

Figure 5 shows that the integrated flux within a given aperture follows a power law with a dependence (flux) $\propto$ (diameter $)^{0.8}$; if circular symmetry holds, this relation corresponds to a surface brightness which decreases as the (radius) ${ }^{-1.2}$. This powerlaw behavior is not atypical of the nuclei of common galaxies; specifically, the surface brightness of Maffei 1 goes as (radius) - $^{-1.0}$ while that of M31 goes as (radius) ( $^{-0.4}$.

\section{INTERSTELLAR EXTINCTION}

The extinction in the direction of Maffei 2 may be estimated by assuming that the energy distribution shown in figure 4 results from an energy distribution like that of M31 (Sandage, Becklin, and Neugebauer 1969) which has been reddened by interstellar extinction. From the ratio of the visual and $2-\mu$ flux a visual extinction of 6.3 mag is obtained; a value of $6.3 \mathrm{mag}$ is also obtained from the $2.2-$ and $1.6-\mu$ data alone. The dependence of extinction as a function of wavelength has been applied from Whitford (1948) as discussed in Becklin and Neugebauer (1968). In figure 4 the energy distribution of Maffei 2 with 6.3 mag of visual extinction removed is compared to that of M31; it is seen that, under the foregoing assumptions, the extinction in the direction of Maffei 2 cannot deviate by more than \pm 0.2 mag from $A_{V}=6.3$ mag. In view of the patchy nature of the obscuration in this direction, an $A_{V}=6.3 \mathrm{mag}$ is reasonably close to the value, $A_{V}=5.2 \mathrm{mag}$, obtained by Spinrad et al. (1971) who applied the same considerations to similar data of Maffei 1. Moreover, both values seem reason- 
able in view of the low galactic latitude, $b=-0.5$, of the Maffei galaxies. However, Kohoutek and Haug (1972) obtained $A_{V}=3.1 \pm 0.4 \mathrm{mag}$ in the direction of the Maffei galaxies. These authors measured photographic $U B V$ colors of stars within a circle of $1^{\circ}$ radius centered on Maffei 1 . The measured colors of these stars were then unreddened by applying the normal trajectory in the $(B-V, U-B)$ diagram. On so doing, they found 111 stars within the magnitude interval $8 \mathrm{mag}<V \leq 15.5 \mathrm{mag}$ with intrinsic colors $(U-B)_{0}=-0.6 \mathrm{mag}$; many of these probably are ordinary B-type stars. Kohoutek and Haug assumed that these stars were on the main sequence; they then used their derived intrinsic luminosities and extinction values to plot $A_{V}$ against distance. They found a rapid increase in $A_{V}$ up to $1.5 \mathrm{kpc}$ but no discernible increase between 2 and $7 \mathrm{kpc}$.

We have been unable to resolve the discrepancy between the two values of $A_{V}$ in the direction of the Maffei galaxies. The patchiness of the extinction in this area of the sky presents an obvious difficulty in Kohoutek and Haug's procedure; stars with below average obscuration tend to be selected. If Kohoutek and Haug's value is correct, then either the Whitford reddening law does not apply in this direction, or both Maffei galaxies have a large red and infrared excess. The former possibility is unlikely in view of the fact that the Whitford extinction law apparently applies even to the very heavily obscured galactic center (Becklin and Neugebauer 1968). Both galaxies, particularly Maffei 1, are unaccompanied by any other evidence for unusual nonthermal activity in the nucleus; specifically there is no evidence, out to 2.2 and probably to $3.5 \mu$, for a dependence of the color with aperture in either galaxy. As will be seen in $\S \mathrm{IV}$, the adopted values of $A_{V}$ play a considerable role in our interpretation of the data on Maffei 1 . Consequently it is important that the discrepancy be understood.

\section{THE NATURE AND DISTANCE OF MAFFEI 2}

Extensive radiofrequency observations of Maffei 2 have been reported since the initial high-frequency detection by Bell, Seaquist, and Braun (1971). The galaxy has been observed in the $21 \mathrm{-cm}$ line by Bottinelli et al. (1971), by Shostak and Weliachew (1971), and by Love (1972). The derived fractional content of hydrogen and the mass derived from the $21-\mathrm{cm}$ rotation curve are consistent with Maffei 2 being a normal $\mathrm{Sb}$ galaxy at a distance of a few Mpc. Love's uncorrected radial velocity for the center of mass of Maffei 2 is $-10 \pm 15 \mathrm{~km} \mathrm{~s}^{-1}$; this is in very good agreement with our optical value for the nucleus which was quoted in $\S \mathrm{II} b$.

Detailed radio-continuum maps of Maffei 2 have been made by Love at $1422 \mathrm{MHz}$ and by Allen and Raimond (1972) who used the Westerbork synthesis array at 1415 $\mathrm{MHz}$. The main features of these radio continuum maps correspond to prominent optical features on our $\mathrm{H} \alpha$ photograph (fig. 3). Love's source $\mathrm{C}$ is the optical nucleus, while his radio component $\mathrm{D}$ corresponds to the prominent outer line of $\mathrm{H}$ if regions designated 1, 1a, and 2 in figure 3. Radio component $\mathrm{B}$ coincides with another $\mathrm{H}$ II region complex on the east side of Maffei 2. Component $F$ may correspond to a faint outer $\mathrm{H}$ II region marked 3 on our photograph. The detailed agreement between radiocontinuum features and optical spiral arms in Maffei 2 resembles that found in other spiral galaxies-particularly in the beautiful Westerbork map of M51 (Mathewson, van der Kruit, and Brouw 1972).

In Spinrad et al. (1971) the distance to Maffei 1 was estimated to be about $1 \mathrm{Mpc}$. The distance to Maffei 2 may best be estimated from the available optical observations using a comparison of the diameters of the largest $\mathrm{H}$ II regions with those in other galaxies of known distance and similar morphological type. In table 2 the angular diameters of the six largest $\mathrm{H}$ II regions visible on figure 3 of Maffei 2 are listed. The angular diameter of the largest $\mathrm{H}_{\text {II }}$ region, No. 4 , is $D_{L}=16^{\prime \prime}$. while the average 
TABLE 2

The Angular Sizes of H iI Regions in Maffei 2

\begin{tabular}{|c|c|}
\hline Region No. & $\begin{array}{c}D^{*} \\
\text { (arc seconds) }\end{array}$ \\
\hline 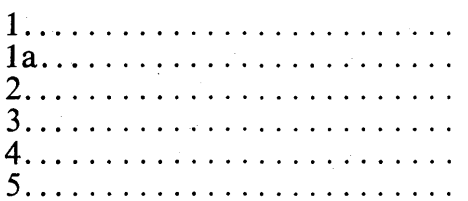 & $\begin{array}{r}12 \\
3 \\
6 \\
2 \\
16 \\
9\end{array}$ \\
\hline
\end{tabular}

* Average measures of Spinrad and Smith.

diameter of the first five $\mathrm{H}$ II regions in Maffei 2 is $D_{\langle 5\rangle}=8^{\prime \prime}$. In table 3 these data are compared with similar data for M31, M33, and NGC 2403; the morphological types of the galaxies are also given. In using diameters of $\mathrm{H}$ il regions to obtain relative distances it is critical to compare galaxies of similar morphological type since $D_{L}$ and $D_{\langle 5\rangle}$ depend on both Hubble type and luminosity class (Sersic 1958; Sandage 1973). Since we are uncertain as to the precise morphological type of Maffei 2, we have used the limited data in table 3 in unweighted form. Further measurement of $\mathrm{H} \mathrm{II}$ region sizes in Sbc II galaxies would be very helpful. Giving the values obtained from $D_{L}$ and from $D_{\langle 5\rangle}$ equal weights, we find that the distance to Maffei 2 is $5 \mathrm{Mpc}$. This estimate is probably uncertain by at least 50 percent, so we adopt $R=5 \pm 2 \mathrm{Mpc}$ for the rest of this paper.

The foregoing estimate of the distance of Maffei 2 is in reasonable agreement with the various estimates obtained by the radiofrequency observers. Thus Bottinelli et al. (1971) used four methods to estimate $R=2.7 \mathrm{Mpc}$. There is a large uncertainty in this value since the four methods give (for an assumed type Sbc) values of $R$ from 1.8 to $10 \mathrm{Mpc}$. Love (1972) has reworked the calculations of Bottinelli et al. (1971) using his data and obtains a distance to Maffei 2 of "about $4 \mathrm{Mpc."} \mathrm{Allen} \mathrm{and}$ Raimond (1972) postulated a physical similarity between Maffei 2 and the galaxies NGC 6946, M51, and NGC 5263 based on their similarity in radio surface brightness. They then compared both the linear sizes and the radio power of the disks of these galaxies with the same quantities for Maffei 2; both methods gave the same distance of about $5 \mathrm{Mpc}$ for Maffei 2.

Both radio and optical observations thus indicate that Maffei 2 is about $4-5 \mathrm{Mpc}$ distant; this is much larger than the distance of $1 \mathrm{Mpc}$ found by Spinrad et al. (1971) for Maffei 1 . The two galaxies have a projected separation of only $45^{\prime}$; are they at the same distance? The distance to Maffei 1 was estimated by two dynamical arguments.

TABLE 3

H il Regions in Sb and Sc Galaxies

\begin{tabular}{cccccl}
\hline \hline Galaxy & $\begin{array}{c}\text { Type and } \\
\text { Luminosity Class }\end{array}$ & $\begin{array}{c}D_{L} \\
\text { (arc seconds) }\end{array}$ & $\begin{array}{c}D_{\langle 5\rangle} \\
\text { (arc seconds) }\end{array}$ & $\begin{array}{c}r \\
(\mathrm{Mpc})\end{array}$ & Reference \\
\hline Maffei $2 \ldots \ldots$ & Sbc II: & 16 & 8 & & This paper \\
M31*....... & Sb I-II & 170 & 100 & 0.7 & Arp (unpublished) \\
M33 $\ldots \ldots \ldots$ & Sc III & 75 & 60 & 0.7 & Sandage 1962 \\
NGC $2403 \ldots$ & Sc III & 15.6 & 11.5 & 3.2 & Sandage 1962 \\
\hline
\end{tabular}

* Only high-surface-brightness $\mathrm{H}$ il regions were used, to be compatible to the obscured Maffei 2 situation. 
These employed the estimated radial-velocity dispersion of the stars in the nucleus of Maffei $1, \sigma_{R}=200 \mathrm{~km} \mathrm{~s}^{-1}$, the estimated rotation velocity near the center, and the effective radius measured from the light distribution. These first two quantities yield a mass estimate for Maffei 1 which is proportional to its distance. Then the observed total luminosity of Maffei 1 yields a mass estimate from an assumed mass-to-light ratio which depends on the distance squared. The value of $1 \mathrm{Mpc}$ for the distance was obtained by putting these two masses equal. A second distance estimate, which does not depend on the luminosity of Maffei 1, was made from $\sigma_{R}$ and the rotation velocity. It also led to a distance of $1 \mathrm{Mpc}$.

If Maffei 1 is at a distance of $1 \mathrm{Mpc}$, then with the derived value of the interstellar extinction, $A_{V}=5.2 \mathrm{mag}$, it has an absolute magnitude $M_{V}=-19.0$. Oort (1971) pointed out that the lack of any observable radio emission is somewhat unusual for such a giant elliptical; however, if Maffei 1 is at a distance of $4 \mathrm{Mpc}$, then it has $M_{V}=-22.0$. This is still within reason for a giant $\mathrm{E}$ galaxy, but the lack of observable radio emission becomes even more puzzling.

Maffei 1 and Maffei 2 also have very similar radial velocities - the optical values are $+165 \pm 50$ and $+173 \pm 20 \mathrm{~km} \mathrm{~s}^{-1}$, respectively, after correction for Galactic rotation. This implies that they have the same distance. The fact that the radial velocities are too close for gravitationally bound systems with these estimated masses need not necessarily be an embarrassment. The plane of their mutual orbit could lie close to the plane of the sky, as Bottinelli et al. (1971) noted.

An additional objection to the idea that Maffei 1 is a supergiant elliptical galaxy stems from the small value of the internal velocity dispersion $\sigma_{R}=200 \mathrm{~km} \mathrm{~s}^{-1}$ which was quoted by Spinrad et al. (1971). Although data on this quantity are sparse, there is evidence that $\sigma_{R}$ increases from around $100 \mathrm{~km} \mathrm{~s}^{-1}$ for dwarf ellipticals like M32 up to about $500 \mathrm{~km} \mathrm{~s}^{-1}$ for supergiants like M87 (Minkowski 1961).

In summary, it seems reasonably well established both from radio and optical observations that Maffei 2 is a galaxy of morphological type near Sbc II which lies at a distance of about $5 \mathrm{Mpc}$, placing it well beyond the Local Group of galaxies and perhaps in the Ursa Major-Camelopardis cloud as Bottinelli et al. (1971) have suggested. The radio and optical distance estimates to Maffei 2 are both almost independent of the assumed value for the interstellar extinction. The proximity of Maffei 1 and Maffei 2 on the plane of the sky, together with the fact that their radial velocities are very similar, could imply that the two galaxies are at the same distance. With a visual extinction $A_{V}=5.2 \mathrm{mag}$, Maffei 1 has an absolute magnitude $M_{V}=-22$ and is a supergiant elliptical if it is $4 \mathrm{Mpc}$ distant. The absence of detectable radio emission and the low value of the internal velocity dispersion are both arguments against Maffei 1 being such a supergiant galaxy.

There seem to be two possible ways of resolving the problem. One unlikely way is to suppose that Maffei 1 really is $4 \mathrm{Mpc}$ distant and that our value of the extinction is too high. If we adopt the value $A_{V}=3.1 \mathrm{mag}$ proposed by Kohoutek and Haug (1972), then Maffei 1 has $M_{V}=-20.1$ if it is 4 Mpc distant. Moreover, with this value of the extinction, the distance estimate obtained by Spinrad et al. (1971) from an assumed mass-to-light ratio (which is probably the more accurate of the two methods they employed) of $1 \mathrm{Mpc}$ should be increased to $2.4 \mathrm{Mpc}$. If, on the other hand, we insist that $A_{V}=5.2 \mathrm{mag}$ for Maffei 1, then it seems inevitable, for the reasons given, to conclude that it is much closer than Maffei 2 and that their proximity on the plane of the sky and their similar radial velocity are both fortuitous.

As a result of these considerations the problems of the nature of Maffei 1 and of its relationship to Maffei 2 are left in an unsatisfactory state. In order to make progress we must resolve somehow the discrepancy between the two ways of obtaining the interstellar extinction. Furthermore, the original dynamical estimate of the distance of Maffei 1 needs to be checked with the aid of higher-quality spectrograms. 
We thank Drs. A. Toomre, I. King, A. Sandage, and H. C. Arp for valuable discussion. The infrared work was supported in part by National Aeronautics and Space Administration grants NGL 05-002-207 and NGL 05-002-007.

\section{REFERENCES}

Allen, R. T., and Raimond, E. 1972, Astr. and Ap. (in press).

Becklin, E. E., and Neugebauer, G. 1968, Ap. J., 151, 145.

Bell, M. B., Seaquist, E. R., and Braun, L. D. 1971, Ap. J. (Letters), 161, L13.

Bottinelli, L., Charnaraux, P., Gerard, E., Gougnenheim, L., Heichmann, J., Kazes, I., and Laugue, R. 1971, Astr. and Ap., 12, 264.

Kohoutek, L., and Haug, U. 1972, Nature Phys. Sci., 236, 44.

Love, R. 1972, Nature, 235, 53.

Maffei, P. 1968, Pub. A.S.P., 80, 618.

Mathewson, R. S., Kruit, P. C. van der, and Brouw, W. N. 1972, Astr. and Ap., 17, 468.

Minkowski, R. L. 1961, Problems of Extragalactic Research, I.A.U. Symposium no. 15, ed. G. C. McVittie (New York: Macmillan Co.).

Oort, J. H. 1971, Nature, 230, 103.

Sandage, A. R. 1962, in Problems of Extragalactic Research, I.A.U. Symposium no. 15, ed. G. C. McVittie (New York: Macmillan Co.).

- 1973 (in preparation).

Sandage, A. R., Becklin, E. E., and Neugebauer, G. 1969, Ap. J., 157, 55.

Sersic, J. L. $1960, Z_{s}$. f. Ap., 50, 168.

Shostak, G. S., and Weliachew, L. 1971, Ap. J. (Letters), 169, L71.

Spinrad, H. 1966, Ap. J., 145, 195.

Spinrad, H., Sargent, W. L. W., Oke, J. B., Neugebauer, G., Landau, Robert, King, Ivan R., Gunn, James E., Garmire, Gordon, and Dieter, Nannielou H. 1971, Ap. J. (Letters), 163, L25.

Whitford, A. E. 1948, Ap. J., 107, 102. 


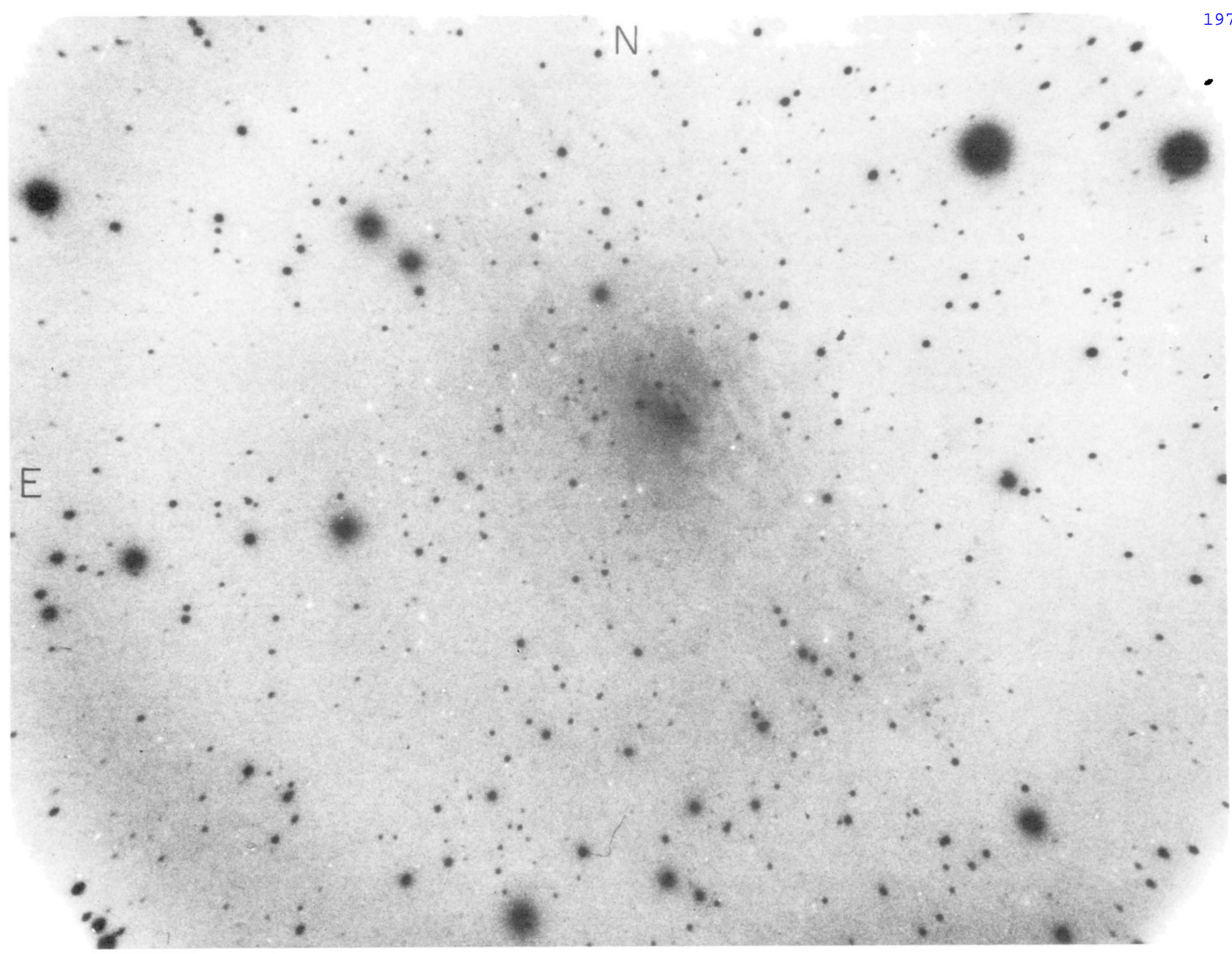

FIG. 1.-An infrared region direct photograph of Maffei 2 obtained by Gunn at the prime focus of the Hale reflector. The image intensifier was an ITT 4708 tube behind a Wratten $89 \mathrm{~B}$ filter. The edges of the field are somewhat vignetted with this system.

SPINRAD et al. (see page 351) 


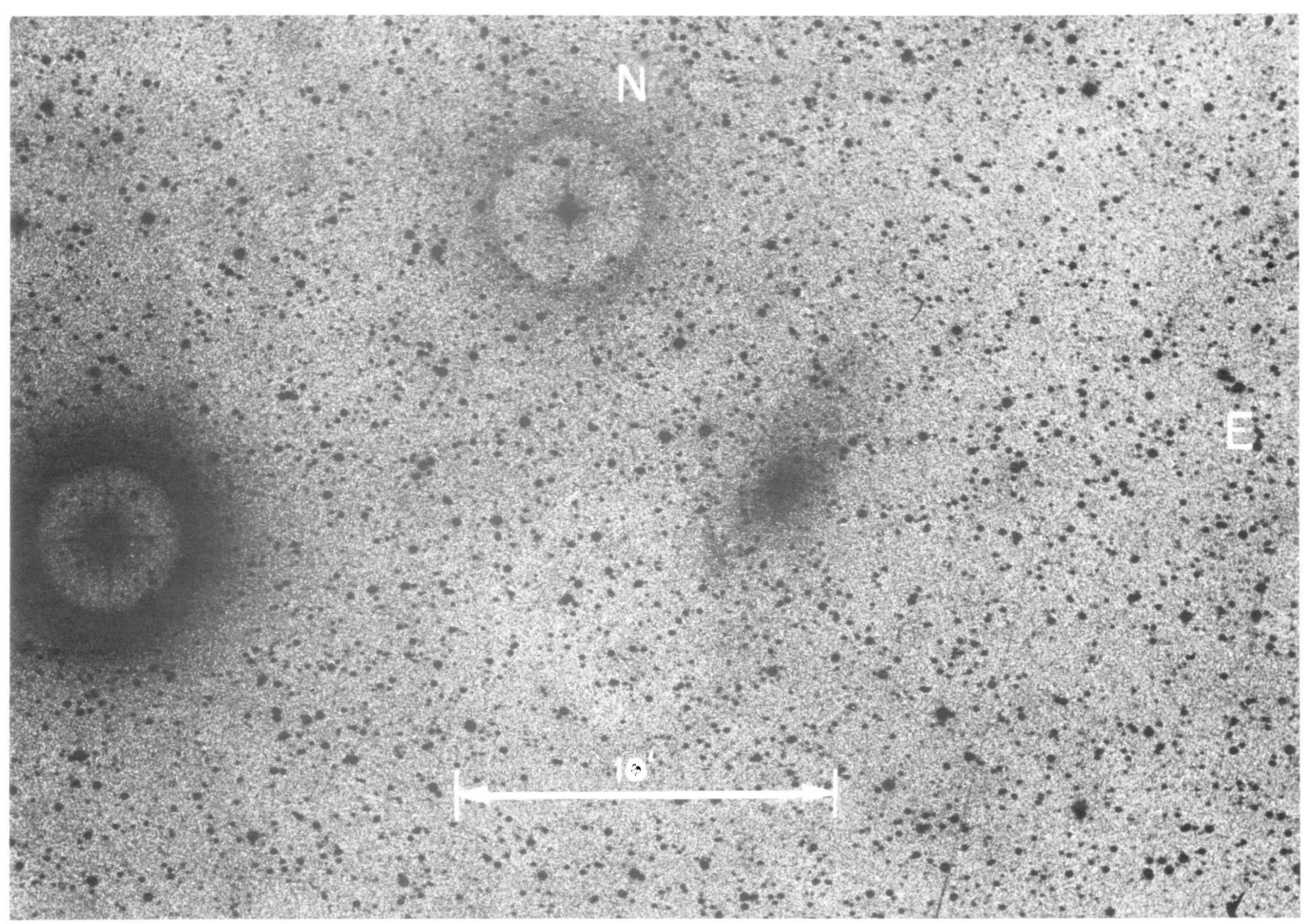

FIG, 2-An enlarged portion of an infrared 48-inch Schmidt plate by Bahcall and Spinrad. The exposure was $3^{\mathrm{h}} 24^{\mathrm{m}}$ on hypersensitized I-N emulsion behind a Wr 89B filter. Note the barlike structure of the inner portions of Maffei 2 and the spurlike arm to the SW. The bright star to the west of Maffei 2 is the red visible star GP Cas.

SPINRAD et al. (see page 352) 




